University of Nebraska - Lincoln

DigitalCommons@University of Nebraska - Lincoln

2008

\title{
Assignment of Polarization-Dependent Peaks in Carbon K-Edge Spectra from Biogenic and Geologic Aragonite
}

\author{
Dong Zhou \\ University of Wisconsin-Madison \\ Rebecca A. Metzler \\ University of Wisconsin-Madison \\ Tolek Tyliszczak \\ Lawrence Berkeley National Laboratory \\ Jinghua Guo \\ Lawrence Berkeley National Laboratory \\ Mike Abrecht \\ Synchrotron Radiation Center \\ See next page for additional authors
}

Follow this and additional works at: https://digitalcommons.unl.edu/usdoepub

Part of the Bioresource and Agricultural Engineering Commons

Zhou, Dong; Metzler, Rebecca A.; Tyliszczak, Tolek; Guo, Jinghua; Abrecht, Mike; Coppersmith, Susan N.; and Gilbert, P. U. P. A., "Assignment of Polarization-Dependent Peaks in Carbon K-Edge Spectra from Biogenic and Geologic Aragonite" (2008). US Department of Energy Publications. 49.

https://digitalcommons.unl.edu/usdoepub/49

This Article is brought to you for free and open access by the U.S. Department of Energy at DigitalCommons@University of Nebraska - Lincoln. It has been accepted for inclusion in US Department of Energy Publications by an authorized administrator of DigitalCommons@University of Nebraska - Lincoln. 


\section{Authors}

Dong Zhou, Rebecca A. Metzler, Tolek Tyliszczak, Jinghua Guo, Mike Abrecht, Susan N. Coppersmith, and P. U. P. A. Gilbert 


\title{
Assignment of Polarization-Dependent Peaks in Carbon K-Edge Spectra from Biogenic and Geologic Aragonite
}

\author{
Dong Zhou, ${ }^{\dagger}$ Rebecca A. Metzler, ${ }^{\dagger}$ Tolek Tyliszczak,,${ }^{\ddagger}$ Jinghua Guo, ${ }^{\ddagger}$ Mike Abrecht, ${ }^{\S}$ \\ Susan N. Coppersmith, ${ }^{\dagger}$ and P. U. P. A. Gilbert ${ }^{*}, \dagger$ \\ Department of Physics, University of Wisconsin-Madison, 1150 University Avenue, Madison, \\ Wisconsin 53706, Advanced Light Source, Lawrence Berkeley National Laboratory, Berkeley, California 94720, \\ and Synchrotron Radiation Center, 3731 Schneider Drive, Stoughton, Wisconsin 53589
}

Received: April 12, 2008; Revised Manuscript Received: July 6, 2008

\begin{abstract}
Many biominerals, including mollusk and echinoderm shells, avian eggshells, modern and fossil bacterial sediments, planktonic coccolithophores, and foraminifera, contain carbonates in the form of biogenic aragonite or calcite. Here we analyze biogenic and geologic aragonite using different kinds of surface- and bulksensitive X-ray absorption near-edge structure (XANES) spectroscopy at the carbon K-edge, as well as highresolution scanning transmission X-ray microscopy (STXM). Besides the well-known main $\pi^{*}$ and $\sigma^{*}$ carbonate peaks, we observed and fully characterized four minor peaks, at energies between the main $\pi^{*}$ and $\sigma^{*}$ peaks. As expected, the main peaks are similar in geologic and biogenic aragonite, while the minor peaks differ in relative intensity. In this and previous work, the minor peaks appear to be the ones most affected in biomineralization processes, hence the interest in characterizing them. Peak assignment was achieved by correlation of polarization-dependent behavior of the minor peaks with that of the main $\pi^{*}$ and $\sigma^{*}$ peaks. The present characterization provides the background for future studies of aragonitic biominerals.
\end{abstract}

\section{Introduction}

The calcium carbonates $\left(\mathrm{CaCO}_{3}\right)$ aragonite and calcite constitute the majority of biominerals, including the outer shells of mollusks: bivalves, gastropods and cephalopods; the endoskeletons of echinoderms, avian eggshells, corals, planktonic coccolithophores and foraminifera, and bacterial biominerals, comprising extant and fossil bacterial biofilms. ${ }^{1,2}$ It is therefore of great interest to characterize all spectral components in X-ray absorption near-edge structure (XANES) spectra from aragonite, which is done here.

One of the most interesting and studied aragonitic biominerals is mother-of-pearl, or nacre: a composite of $95 \%$ aragonite and $5 \%$ organic molecules. ${ }^{1,2}$ Its remarkable toughness-many thousands of times more resistant to fracture than aragonite ${ }^{3}$-has stimulated many studies of nacre's mechanical performance, structure, proteins and glycoproteins, and the role that these might play in nacre formation. ${ }^{4-8}$ We recently discovered that nacre exhibits X-ray linear dichroism, a phenomenon widely studied in man-made materials but never before reported in a biomineral. ${ }^{8,9}$ Dichroism in carbonates generates a polarizationdependent imaging contrast (PIC), which is particularly useful to investigate the meso-scale architecture of nacre using X-ray photoelectron emission spectromicroscopy $(\mathrm{X}-\mathrm{PEEM})^{10}$ and scanning transmission X-ray spectromicroscopy (STXM). ${ }^{11,12}$ With X-PEEM we revealed that the 400-nm high, 5- $\mu \mathrm{m}$ wide aragonite tablets that form the layered abalone nacre structure are arranged in stacks of co-oriented tablets, staggered with respect to each other.

We have previously extensively characterized the origin of PIC and X-ray linear dichroism with a series of pure-

\footnotetext{
* Corresponding author. Previously publishing as Gelsomina De Stasio. E-mail:pupa@physics.wisc.edu.

$\dagger$ University of Wisconsin-Madison.

\$ Lawrence Berkeley National Laboratory.

§ Synchrotron Radiation Center.
}

spectroscopy experiments on macroscopic single crystals of aragonite and calcite while rotating the sample position with respect to the polarization vector in linearly polarized synchrotron light. ${ }^{9}$ High-resolution X-ray diffraction (XRD) and neutron diffraction indicate structural and microstructural differences between biogenic and geologic aragonite; there are differences in atomic positions as well as in the lattice parameters. ${ }^{13-15}$ Specifically, biogenic aragonite is slightly distorted with respect to geologic aragonite. ${ }^{15}$ XANES spectroscopy probes the local electronic environment of carbon, and is therefore not expected to be sensitive to the small structural differences evidenced by XRD.

To confirm this expectation, here we do a direct comparison of spectra from geologic and nacreous aragonite by doing a dichroism experiment on a single aragonite tablet in nacre. This challenging experiment is enabled by two state-of-the-art devices: a high-resolution scanning transmission X-ray microscope (STXM) ${ }^{11}$ and its elliptically polarizing undulator (EPU) source. The STXM enables the identification of a single tablet in a thin section of nacre, and the acquisition of a XANES ${ }^{16}$ spectrum with horizontally polarized X-rays, then the EPU makes it possible to rotate the polarization vector between $0^{\circ}$ and $90^{\circ}$.

To assign all the observed peaks, we also acquired XANES data on geologic aragonite in total electron yield (TEY) and fluorescence yield (FY) modes, as a function of polar angle $\theta$ between polarization vector and aragonite crystallographic $c$-axis. In all experiments we find similar spectra, with the two well-known and documented main peaks also found in carbonate anions deposited on silver ${ }^{16-18}$ as well as many biominerals. ${ }^{8,9,12,16}$ We also find and fully document for the first time that four minor peaks appear in the aragonite XANES spectra, which are not present in carbonate anions deposited on silver. ${ }^{16-18}$ Despite their relevance in biomineralization, the minor peaks in carbonates have received remarkably little attention. 
In the work of Benzerara et al., ${ }^{19}$ the minor peaks are not relevant to the experiment described, which focuses mostly on organic peaks, therefore the authors do not mention them. In their spectra, however, the minor peaks dramatically differ in intensity between intracellular and extra-cellular regions of microbialites (see Figure $4 \mathrm{~d}$ in ref 19). The intracellular aragonite can be considered akin to biogenic aragonite as explained in the discussion, while the extra-cellular aragonite is akin to geologic aragonite. In the 2008 work of Metzler et al., ${ }^{7}$ similar minor peaks in calcite are the ones most affected by peptide and protein binding. Specifically, two minor peaks at $\sim 296$ and $\sim 298 \mathrm{eV}$, assigned to $\mathrm{C} 1 \mathrm{~s} \rightarrow \sigma^{*}$ in calcite $\mathrm{CO}$ bonds, were suppressed when bonds were formed with carboxyl groups in acidic amino acids (aspartate and glutamate) and remained unaltered in control experiments, in which these amino acids were substituted with nonbinding aspargine and glutamine. See Figures 5-8 in ref 7 for minor-peak suppression upon binding, and Figure 7 in ref 7 for the controlled experiment on calcite-binding and nonbinding peptides. From these two experiments on natura ${ }^{19}$ and synthetic ${ }^{7}$ biominerals, we conclude that the minor carbonate peaks are extremely relevant in biomineralization. They therefore deserve further investigation. The main goal of the present work is to assign the minor peaks to $\mathrm{CO}$ bonds in bulk aragonite, and to deduce from their polarization dependence that all bulk aragonite peaks are divided into two groups of vector-type bonds, perpendicular to each other.

\section{Experimental Methods}

Sample Preparation. In our experiments, the biogenic aragonite samples are from red abalone (Haliotis rufescens) shells purchased from Sea Shell City (Fenwick Island, DE) that originated from an abalone farming facility. The geologic aragonite samples originated from Sefrou, Morocco.

Biogenic Aragonite Thinning by Focused Ion Beam (FIB). One red abalone nacre sample with the growth direction approximately parallel to the milled sample surface was first sputter-coated with $\sim 20 \mathrm{~nm} \mathrm{Au}$, then milled down to $\sim 80$-nm thickness with an FIB using a Zeiss 1500XB CrossBeam Workstation at the Materials Science Center, UW-Madison. After FIB milling, the layered structure of nacre is retained and conspicuous, as shown below.

Biogenic Aragonite Thinning by Tripod Polishing. Another red abalone nacre piece, with the growth direction $\sim 21^{\circ}$ from the normal to the polished sample surface, was glued (Loctite 460 super glue) onto a sample holder, polished with Allied TechPrep polishing wheel using the Multiprep sample holder assembly. The polishing angle was $2^{\circ}$. At the end, the sample was immersed in acetone for $1 \mathrm{~h}$ to dissolve the glue and separate the sample from its holder. From the STXM absorption spectrum, the thickness of the thinnest region was estimated to be about $250 \mathrm{~nm}$.

Geologic Aragonite. Three geologic aragonite samples were chiseled from one large aragonite crystal from Sefrou, Morocco. They were then cleaned, first in trichloroethane, then in acetone, and finally in ethanol ultrasonic baths, for $20 \mathrm{~min}$ each. This procedure should have removed any organic contamination from the surface of minerals. We detected, however, peaks corresponding to organic materials. Carbonates are notorious absorbers of oil and other organic humic substances. Some organic contamination in geologic aragonite, therefore, is unavoidable.

For the STXM experiment on beamline 11.0.2 at ALS, the aragonite crystal was crushed by mortar and pestle. The

\section{STXM, front view}
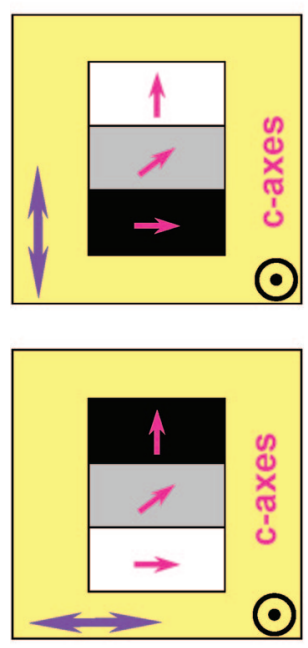

TEY XANES ON HERMON skewed view

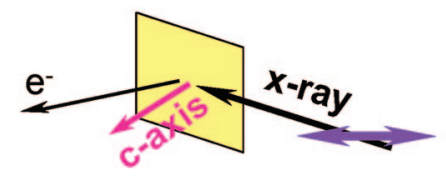

TEY and FY XANES on BL7 front view

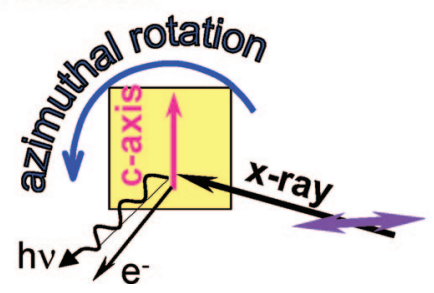

Figure 1. Schematic of the three experimental approaches used in this work: STXM of bio- and geo-aragonite, XANES spectroscopy of geo-aragonite done both in TEY on the HERMON beamline, and in TEY and FY on beamline 7. In all cases, the crystal $c$-axis of bio- or geo-aragonite crystals is indicated by magenta arrows, the linear polarization vector by purple arrows, and the X-ray beam by black arrows in XANES spectroscopy, or by a bull's eye circle in STXM, as the beam is perpendicular to the sample surface. All three experiments provide polarization dependence, with higher intensity of the $\pi^{*}$ peak when the aragonite crystal $c$-axis is parallel to the polarization $\left(\theta=0^{\circ}\right)$, and minimum when perpendicular $\left(\theta=90^{\circ}\right)$. In the STXM panel, we show three $c$-axes in plane, although these could be off-plane, as often happens with microscopic biogenic crystals of unknown orientations. In STXM we also show that the polarization from the EPU can be rotated by $90^{\circ}$, and this changes the $\mathrm{C} \pi^{*}$ peak intensity in aragonite crystal spectra and images, as indicated here by their gray-levels (white when $\theta=0^{\circ}$, black when $\theta=90^{\circ}$, gray when intermediate). In the XANES spectroscopy experiment on HERMON the polarization is fixed, and the sample position is fixed, with the $c$-axis normal to the crystal surface. On beamline 7 the polarization is fixed and horizontal, but the macroscopic single crystal of geo-aragonite, with the $c$-axis in plane, can be rotated azimuthally (around its normal), thus enabling the full characterization of polar dependence, that is, peak intensity as a function of $\theta$. Both TEY and FY were done in the same configuration, and acquired simultaneously.

aragonite powder was suspended in a slurry, deposited on a $\mathrm{Si}_{3} \mathrm{~N}_{4}$ window, and air-dried.

For the macroscopic XANES spectroscopy experiments on beamline 7.0.1 at ALS and HERMON at SRC, the geologic aragonite samples were embedded in epoxy (Epoxicure resin and hardener, Buehler, Lake Bluff, IL) so that their c-axes were either in-plane or perpendicular to the polished sample surface. Once cured, the blocks were polished with decreasing size alumina grit down to $0.05 \mu \mathrm{m}$ (Masterprep, Buehler, Lake Bluff, IL). For the in-plane sample, the crystal orientation was verified by XRD (Hi-Star 2-D detector, Materials Science Center, UWMadison), and the $c$-axis was confirmed to be in plane with an uncertainty of $\pm 2^{\circ}$. The careful positioning of the crystal sample was crucial to ensure control over the polar angle $\theta$ between the crystal $c$-axis and the photon polarization. The sample was then repolished to remove the top $1000 \mu \mathrm{m}$ of the crystal, as the attenuation length of $8 \mathrm{keV}$ photons from the XRD experiment is $250 \mu \mathrm{m},{ }^{20}$ and that layer was therefore to be considered radiation-damaged surface. Finally the samples were sputter coated with $1 \mathrm{~nm}$ Pt in the area to be analyzed, and 50 $\mathrm{nm} \mathrm{Pt}$ around it, to provide electrical conductivity to the otherwise insulating crystals. ${ }^{21}$ 
A nacre transmission (STXM, EPU $30^{\circ}$ )

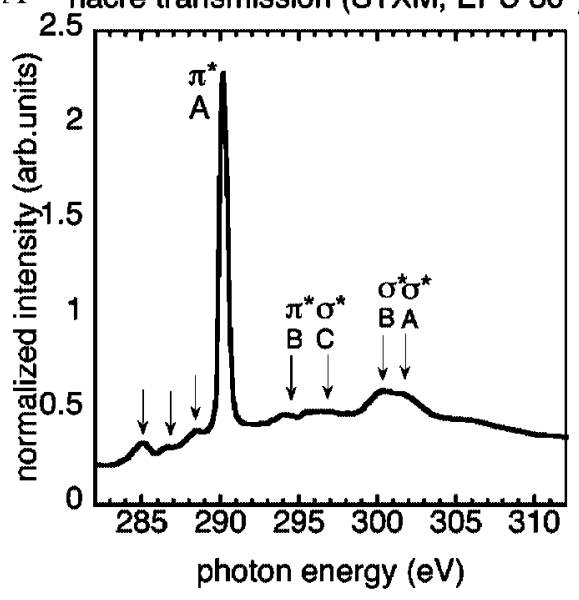

C

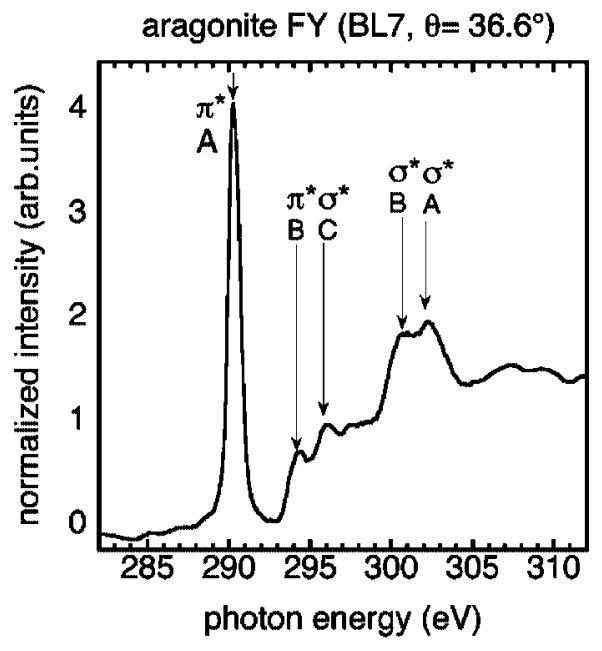

B

aragonite TEY (HERMON, $\theta=26^{\circ}$ )

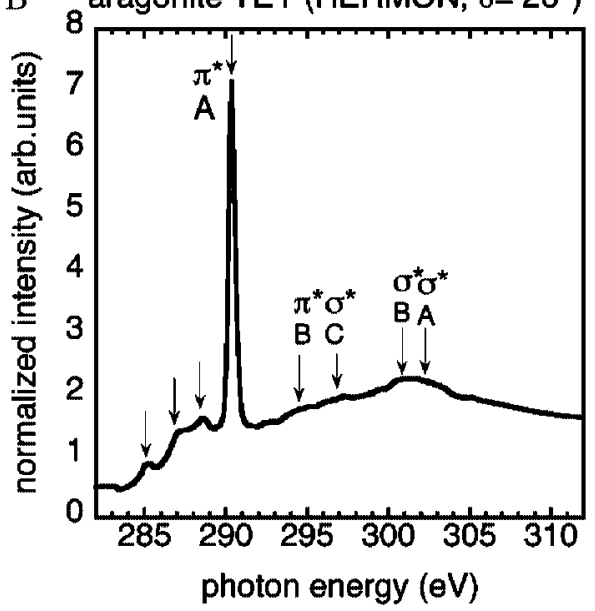

D
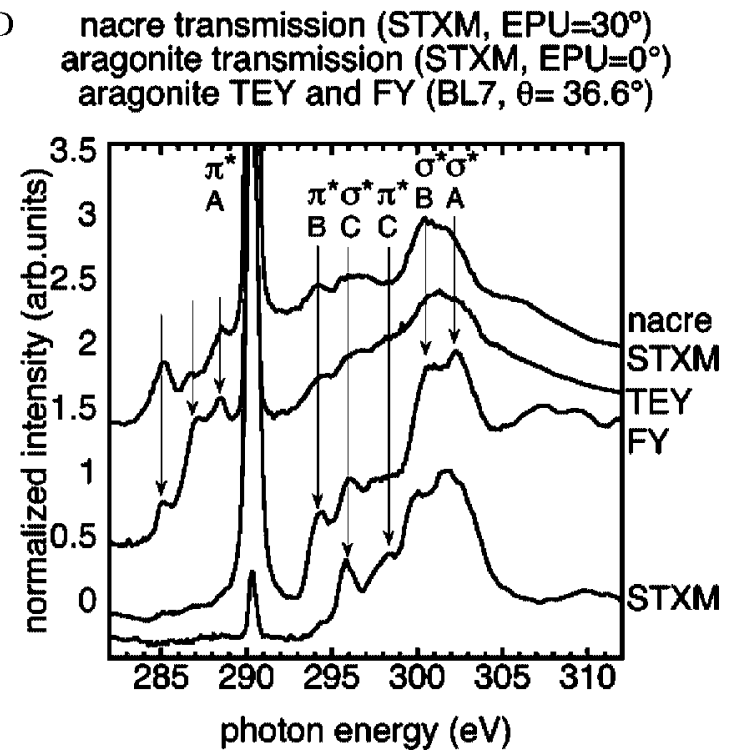

Figure 2. XANES carbon K-edge spectra of biogenic (A) and geologic (B,C) aragonite. (A) XANES spectrum acquired with STXM at the ALS, from a nacre sample, thinned by FIB. This spectrum was taken with the linear polarization from the EPU at an intermediate angle $\left(30^{\circ}\right)$, so the $\pi^{*}$ is neither maximum nor minimum. (B) Aragonite TEY spectrum from the HERMON beamline at SRC, acquired on a single macroscopic crystal of aragonite. The polar angle $\theta$, between the crystal $c$-axis and the photon polarization vector for this spectrum was $\theta=26^{\circ}$. (C) FY from aragonite on beamline 7.0.1 at the ALS, acquired from another macroscopic crystal of aragonite, with the $c$-axis in plane and $\theta=36.6^{\circ}$. (D) Comparison between aragonite TEY spectrum and from ALS Beamline 7, the nacre transmission spectrum in A, and the aragonite FY spectrum in C. FY and TEY on BL7 were acquired simultaneously. The plot also shows at the bottom the STXM spectrum of geologic aragonite, which includes surface and bulk contributions, but, as the FY spectrum, is dominated by the bulk signal. The STXM and FY bulk spectra therefore lack the surface peaks at low energies. All spectra are normalized to the background beamline output curve $\left(I_{0}\right)$, and the pre-edge intensity is set to 0 . All spectra were aligned so the main $\pi^{*}$ peak is at $290.3 \mathrm{eV}$.

\section{Data Acquisition}

STXM on BL 11.0.2. We used the STXM on beamline 11.0.2 at the Advanced Light Source (ALS). The microscope is described in detail elsewhere. ${ }^{11}$ This beamline is equipped with an EPU source, which enables the selection of both linearly and circularly polarized light. We always used linearly polarized light, and rotated the photon polarization vector in a $90^{\circ}$ range. In the STXM experiment, optical density (OD) maps are presented. OD is defined to be the logarithm of the ratio between the transmitted light intensity $(I)$ and incident light intensity $\left(I_{0}\right)$ for a given wavelength $\lambda$ :

$$
O D_{\lambda}=\ln \left(\frac{I_{o}}{I}\right)=n \mu d
$$

OD images, therefore, reflect a combination of concentration $(n)$, absorption coefficient $(\mu)$, and thickness $(d)$ of the sample.
The best experimental conditions are for regions of the sample adjacent to an empty region of space, through which X-ray photons pass unabsorbed to provide a reference intensity $I_{0}$, which is acquired simultaneously with $I$. For images, the brightest pixel in the image is taken as $I_{0}$. For spectra, we measured the average intensity of a bright open region including many pixels, and used this average as $I_{0}$ to avoid artificial enhancement of spectroscopic features in XANES spectra. Hereafter, we only report OD images in which bright pixels represent high concentration of the chemical species with peak absorption at the selected photon energy.

Images taken at off-peak energies reveal mostly sample thickness. On-peak images reveal both thickness and chemical composition. Therefore, images calculated by subtraction of onpeak and off-peak OD images distinguish different chemical components, and are referred to as OD difference maps. Images calculated by subtracting OD images with different polarizations 
reveal the polarization behavior of individual XANES peaks. These OD polarization-dependence maps are acquired at a single photon energy and two EPU polarizations $\left(0^{\circ}\right.$ and $\left.90^{\circ}\right)$. XANES spectra were also acquired by STXM. These were extracted from stacks of images acquired across the entire carbon energy range $(280-320 \mathrm{eV})$, for both the biogenic and geologic aragonite samples.

Change in polarization from the EPU does not affect the flux, the energy calibration, or the spot size. The spot size on 11.0.2. STXM is $20 \mathrm{~nm} \times 20 \mathrm{~nm}(h \times v)$, and images were acquired with 400 pts $\times 400$ pts, over an $8 \mu \mathrm{m} \times 8 \mu \mathrm{m}$ nacre area.

Macroscopic XANES on BL 7.0.1. On beamline 7.0.1 at the ALS, an ultrahigh vacuum chamber is equipped with both a solid-state detector for FY and a picoammeter to measure the TEY simultaneously and from the same sample spot. The sample itself in this chamber can rotate azimuthally by $270^{\circ}$ with an accuracy of less than $1^{\circ}$. One of the geologic aragonite single crystal samples, coated with $1 \mathrm{~nm}$ Pt in the analyzed area, and $50 \mathrm{~nm} \mathrm{Pt}$ around it, ${ }^{21}$ was measured in this chamber, with the $c$-axis in plane, and the azimuthal rotation of the manipulator, producing a varying polar angle $\theta$ between the fixed polarization vector and the crystal $c$-axis. The source of this beamline is an undulator with linearly polarized light fixed in the horizontal direction. The spot size on BL 7 is $100 \mu \mathrm{m} \times 100 \mu \mathrm{m}(h \times v)$.

Macroscopic XANES on HERMON. On the HERMON beamline at SRC, we analyzed another geologic aragonite single crystal, mounted with the $c$-axis perpendicular to the polished sample surface. This sample was also coated with $1 \mathrm{~nm}$ Pt in the analyzed area, and $50 \mathrm{~nm} \mathrm{Pt}$ around it. ${ }^{21}$ In this case, the polar angle $\theta$ was varied by rotation of the sample manipulator around its vertical shaft. The spot size on HERMON is 1000 $\mu \mathrm{m} \times 100 \mu \mathrm{m}(h \times v)$.

Energy Calibration and Spectra Normalization. The energy scale was calibrated once using the $\mathrm{CO}_{2}$ absorption lines on STXM. Aragonite $\pi^{*}$ peaks were detected at $290.3 \mathrm{eV}$, then all other spectra from STXM, BL 7 an HERMON were aligned so their $\pi^{*}$ peaks were at $290.3 \mathrm{eV}$. All spectra were normalized to the background $I_{0}$ curve, and 1 was subtracted so the preedge intensity is zero. We finally rescaled the TEY spectra $(\times 4)$, to make their intensities similar to all other FY and STXM spectra.

\section{Results}

In Figure 1 we present a schematic diagram showing the sample position, the $c$-axis orientation, and the polarization vector in all four experimental approaches used in this work.

In Figure 2 we report a total of five XANES spectra, acquired from biogenic aragonite in nacre and two samples of geologic aragonite. The various spectra were acquired using the three different instruments working in transmission, total yield, and fluorescence, and reveal interesting similarities and differences. First and foremost, all spectra show main peaks at 290.3 and $302.2 \mathrm{eV}$, due to transitions between a carbon core level and an antibonding $\pi^{*}$ molecular orbital, $\mathrm{C} 1 \mathrm{~s} \rightarrow \pi^{*}$, and from a core level to a $\sigma^{*}$ orbital, $\mathrm{C} 1 \mathrm{~s} \rightarrow \sigma^{*}$, respectively. These peaks are labeled $\pi^{*}$-A and $\sigma^{*}$-A in Figure 2 .

In both cases the carbon atom probed contributes to the carbon-oxygen bonds in the carbonate $\mathrm{CO}_{3}{ }^{2-}$ group. Because the formal bond order of the three carbonate $\mathrm{CO}$ bonds is 1.3 and it is ambiguous to assign these peaks to either $\mathrm{C}-\mathrm{O}$ or $\mathrm{C}=\mathrm{O}$, we hereafter refer to all bonds between carbon and oxygen as $\mathrm{CO}$ bonds. Both main $\mathrm{CO}$ bond spectral peaks $\left(\pi^{*}\right.$-A and $\left.\sigma^{*}-\mathrm{A}\right)$ are well-known, predicted by theoretical calculations $^{16}$ and also observed in monolayers of carbonate anions deposited on silver. ${ }^{16-18}$ Many other minor peaks are observed in Figure 2, indicated by arrows, and are most visible in Figure $2 \mathrm{D}$, in which the vertical scale is magnified. All peaks are also reported and assigned to specific bonds in Table 1 . Notice in particular the peaks at 294.2, 295.9, and $298.3 \mathrm{eV}$ (labeled $\pi^{*}$ $\mathrm{B}, \sigma^{*}-\mathrm{C}$, and $\pi^{*}-\mathrm{C}$, for the reasons described below). These peaks had been previously observed in calcite, ${ }^{7}$ aragonite, $, 9,19$ and another unknown $\mathrm{CaCO}_{3},{ }^{22}$ but had never been discussed nor assigned to specific bonds.

The transmission and FY spectra are bulk-sensitive (escape depth $\sim 100 \mathrm{~nm}$ ) while the TEY spectra are surface sensitive $(\sim 3 \mathrm{~nm}) .{ }^{9}$ Transmission and FY spectra are therefore expected to lack any surface-associated spectral features. In Figure 2 the three lower energy peaks at 285.0, 286.7, and $288.4 \mathrm{eV}$ are not present in the bulk-sensitive STXM and FY spectra, and are very pronounced in all the TEY spectra. These peaks are due to $\mathrm{C} 1 \mathrm{~s} \rightarrow \pi^{*}$ in $\mathrm{C}=\mathrm{C}, \mathrm{C} 1 \mathrm{~s} \rightarrow \sigma^{*}$ in $\mathrm{C}-\mathrm{H}$, and $\mathrm{C} 1 \mathrm{~s} \rightarrow \pi^{*}$ in $\mathrm{C}=\mathrm{O}$ in carboxylate. ${ }^{12,23} \mathrm{We}$ interpret the presence of these peaks in TEY spectra as a sign of surface contamination of the aragonite crystals, which, despite many repeated attempts, could not be eliminated. It is possible, but not likely, that these peaks are a result of aragonite crystal surface relaxation, reconstruction, or hydration as discussed by de Leeuw and Parker $^{24}$ for aragonite or by Magdans et al. ${ }^{25}$ for calcite surfaces. In this case, the presence of $\mathrm{C}=\mathrm{C}$ and $\mathrm{C}=\mathrm{O}$ bonds could be understood, but not $\mathrm{C}-\mathrm{H}$. The peak at $286.7 \mathrm{eV}$ is reliably assigned to $\mathrm{C}-\mathrm{H}^{7,22,23}$ and should really not be present in pure aragonite. We therefore conclude that all three peaks originate from organic molecules naturally present in biogenic aragonite and contaminating the geologic aragonite surface.

The STXM spectrum of nacre is similar to TEY aragonite because the FIB-milled sample is extremely thin (estimated 80 $\mathrm{nm}$ from SEM observation, and perhaps thinner), thus the front and back surfaces constitute a considerable proportion of the traversed sample. Other thicker nacre samples (tripod-polished), such as that of Figure 4, exhibit spectra more similar to the thick aragonite STXM spectrum, or the FY spectrum.

In Table 1, notice that the observed minor peaks at 294.2, 295.9, 298.3, and $300.2 \mathrm{eV}$ are from the bulk, not the surface, of aragonite crystals, as they appear in transmission spectra acquired with STXM both in biogenic and geologic aragonite, and in bulk-sensitive FY spectra. In bulk aragonite, $\mathrm{C}$ is only bonded to $\mathrm{O}$ and not to other $\mathrm{C}$ or $\mathrm{Ca}$ atoms, therefore these peaks must be $\mathrm{CO}$ bonds. We therefore assign them to $\mathrm{CO}$ in carbonates, by analogy with other $\mathrm{CO}$ bonds reported previously. ${ }^{12}$ The further assignment of two of these minor peaks to $\mathrm{CO}$ bond $\pi^{*}$ antibonding orbitals and two to $\mathrm{CO}$ bond $\sigma^{*}$ orbitals is explained below. Although $\mathrm{C}-\mathrm{C}$ peaks also occur at these energies, these four peaks cannot be assigned to $\mathrm{C}-\mathrm{C}$ bonds, as such bonds do not occur in aragonite. ${ }^{12,26,27}$ Furthermore, contaminants should not exhibit dependence on polarization angle, as these peaks do. They cannot therefore originate from the organic contaminants responsible for the pre-290 eV peaks.

Interestingly, the minor peaks that were found to be dramatically affected by biomineral formation, ${ }^{7,9,19}$ also appear to be different between biogenic and geologic aragonite in the present work. Compare, for example, the STXM spectra of nacre and aragonite in Figure 2D (top and bottom spectra). The minorpeak intensity differences in these two spectra are in remarkable agreement with those from intracellular and extracellular aragonite, in the work of Benzerara et al. ${ }^{19}$

In Figure 3 we report the STXM data acquired on a nacre sample thinned by FIB. The small nacre region in Figure $3 \mathrm{C}$ 
A

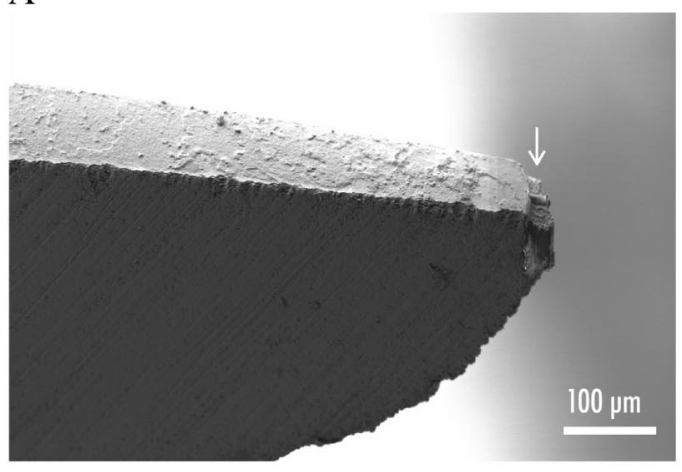

$\mathrm{C}$

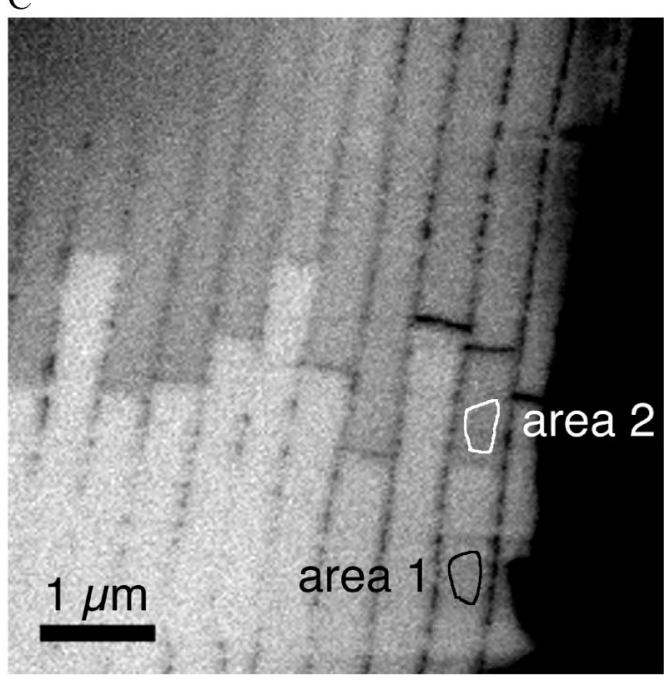

$\mathrm{E}$

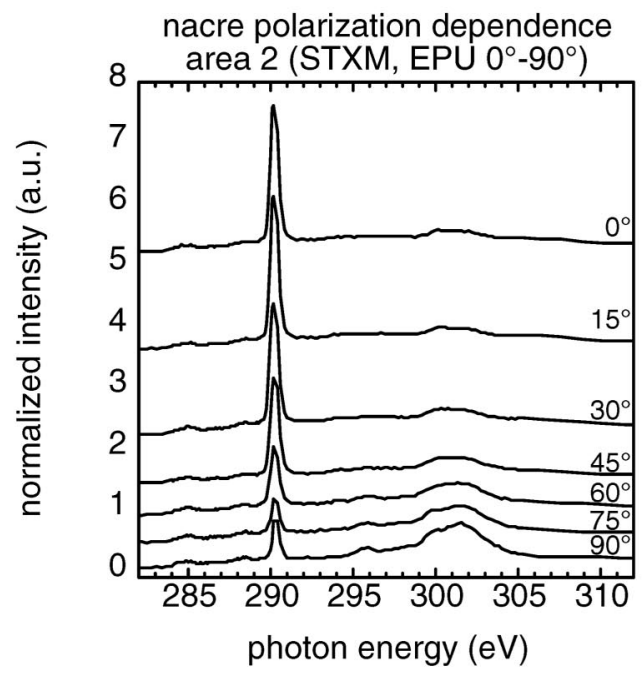

B

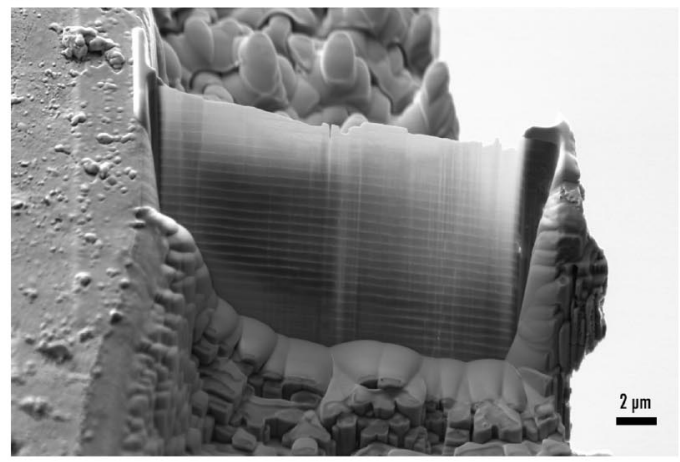

$\mathrm{D}$

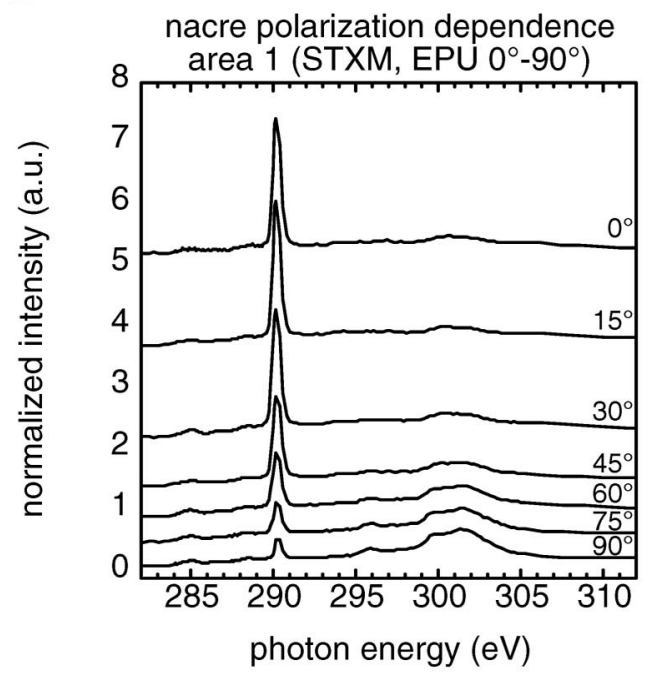

$\mathrm{F}$

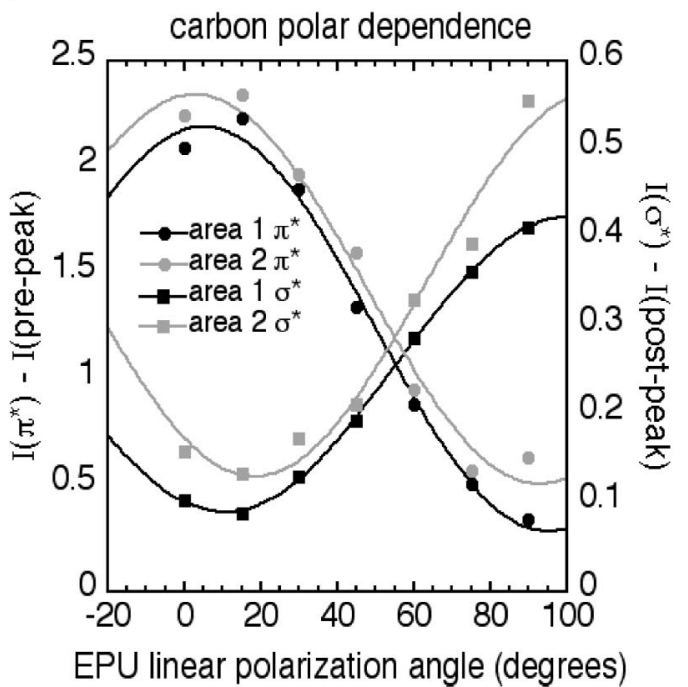

Figure 3. STXM data acquired on a nacre cross-section prepared by FIB. (A) SEM image of the nacre sample at low magnification. (B) SEM image of the same sample at higher magnification. (C) OD difference map from $290.3 \mathrm{eV}$ (carbonate CO bond $\pi^{*}$-A) and $283 \mathrm{eV}$ images. The image is contrast-enhanced for clarity. The areas from which spectra were extracted are outlined in black and white. The nacre growth direction in this sample was in plane and perpendicular to the nacre layers. (D) Carbon spectra from a single aragonite tablet, labeled area 1 in C, acquired with different linear polarization orientations from the EPU. These are not polar angles $\theta$, as defined in Figure $2 \mathrm{~B}$. The $c$-axis direction of microscopic aragonite tablets in nacre is unknown, so the only known value is the direction of photon polarization vector, reported here. (E) Identical series of spectra from another single aragonite tablet, labeled area 2 in panel C. (F) The intensity variation of the main carbonate $\pi^{*}$ and $\sigma^{*}$ peaks are reported as a function of polarization angle. The best data fits are also reported as solid lines. The fitting formula for the $\pi^{*}$-A peak intensity is $I\left(\pi^{*}\right)-I($ pre-edge $)=\alpha \cos \left(\theta+\theta_{0}\right)^{2}+I_{0}$, The fitting formula for the $\sigma^{*}$-A peak intensity is $I\left(\pi^{*}\right)-I($ pre-edge $)=\beta$ sin $\left(\theta+\theta_{0 \mathrm{~b}}\right)^{2}+I_{0 b}$. The pre-peak is defined as the energy position immediately before the $\pi^{*}$-A peak (pre-peak $=289 \mathrm{eV}$ ), while the post-peak is immediately after the $\sigma^{*}$-A peak (post-peak $=306 \mathrm{eV}$ ). The fitting results are as follows: Area $1: \alpha=1.90, \theta_{0}=-5.00, I_{0}=0.29, \beta=0.33, \theta_{0 \mathrm{~b}}=-10.58, I_{0 \mathrm{~b}}=0.09$; Area 2: $\alpha=1.83, \theta_{0}=-2.74, I_{0}=0.51, \beta=0.44, \theta_{0 \mathrm{~b}}=-18.34, I_{0 \mathrm{~b}}=0.13$.

clearly shows individual nacre tablets, arranged in almostvertical layers. These tablets form two distinct stacks with different orientations of the aragonite crystal $c$-axes. The orientations are identified by two different gray levels. This is 
A

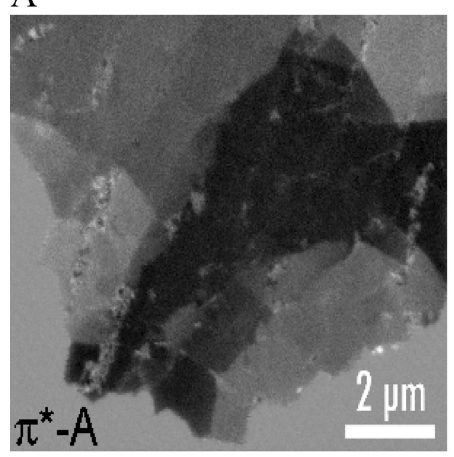

$\mathrm{D}$

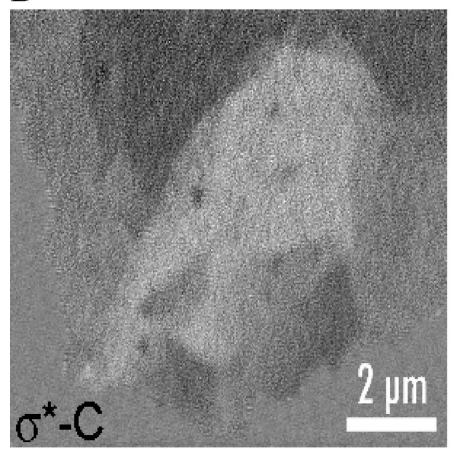

B

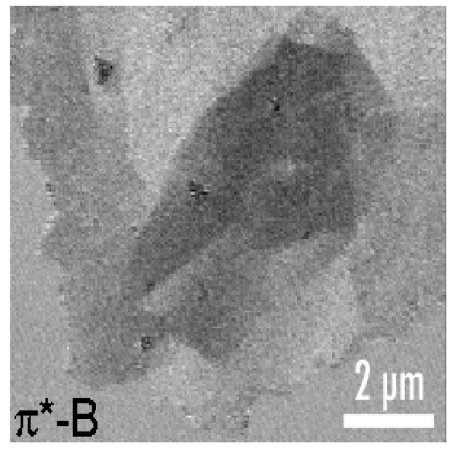

$\mathrm{E}$

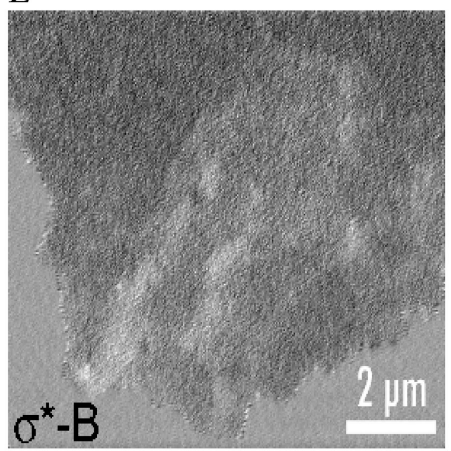

$\mathrm{C}$

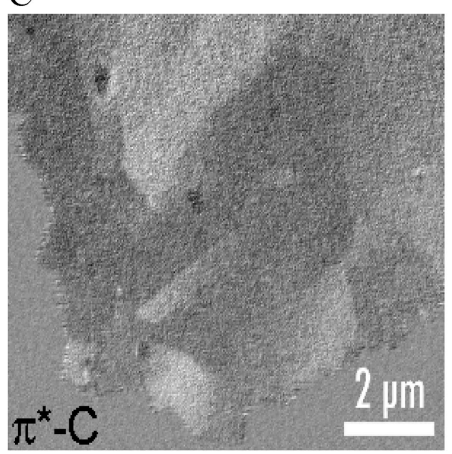

$\mathrm{F}$

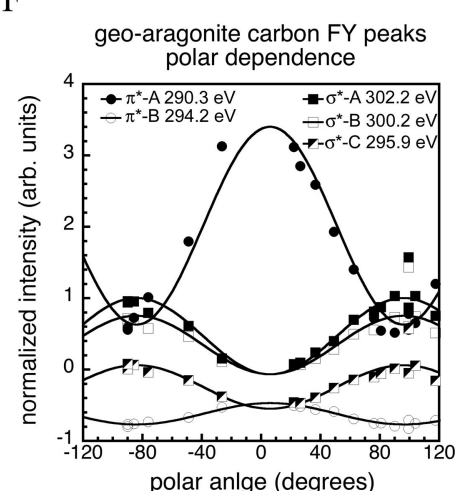

Figure 4. Polarization-dependence maps of the carbon peaks in aragonite. The sample is red abalone nacre thinned by tripod polishing into a $2^{\circ}$-wedge shape. The thickness is larger at the top of the image and minimum at the bottom of the image (estimated from absorption to be approximately $250 \mathrm{~nm}$ ). The nacre growth direction in this sample was approximately $21^{\circ}$ from the normal to the sample surface. These maps are differences of two OD images, acquired with vertical and horizontal polarizations, respectively. The polarization-dependence maps were acquired on five different carbon peaks. Specifically, (A) OD polarization-dependence map of carbonate $\pi^{*}$ peak at $290.3 \mathrm{eV}\left(\pi^{*}\right.$-A); (B) OD polarization-dependence map at $294.2 \mathrm{eV}\left(\pi^{*}-\mathrm{B}\right)$; (C) OD polarization-dependence map at $298.3 \mathrm{eV}\left(\pi^{*}-\mathrm{C}\right)$; (D) OD polarization-dependence map at $295.9 \mathrm{eV}\left(\sigma^{*}-\mathrm{C}\right)$; (E) OD polarization-dependence map of carbonate $\sigma^{*}$ at $300.2 \mathrm{eV}\left(\sigma^{*}-\mathrm{B}\right)$. Polarization-dependence map A is much more intense than all the other maps, thus it shows greater detail and more gray levels, corresponding to different crystal orientations. The other maps show fewer gray levels, due to lower peak intensity. Map E shows a much weaker signal, but is clearly opposite to A, as expected. The contrast in polarization-dependence maps $\mathrm{A}, \mathrm{B}$, and $\mathrm{C}$ is similar, and inverted with respect to D and $\mathrm{E}$. We interpret this result as indicative of CO bonds $\pi^{*}$ orbitals for $\mathrm{A}$, B, and $\mathrm{C}$, and $\mathrm{CO} \sigma^{*}$ for D and E. (F) Polar dependence curve obtained with FY from a macroscopic single crystal of geo-aragonite (beamline 7). Notice the correlation of the main $\pi^{*}$ at $290.3 \mathrm{eV}$ and the $294.2 \mathrm{eV}$ peak (both are circles) and their anticorrelation with the other three peaks $295.9 \mathrm{eV}, 300.2$ and $302.2 \mathrm{eV}$ peaks (squares). These polar dependence curves corroborate the correlations in the polarization-dependence maps in A and B, and in $\mathrm{D}$ and $\mathrm{E}$.

A

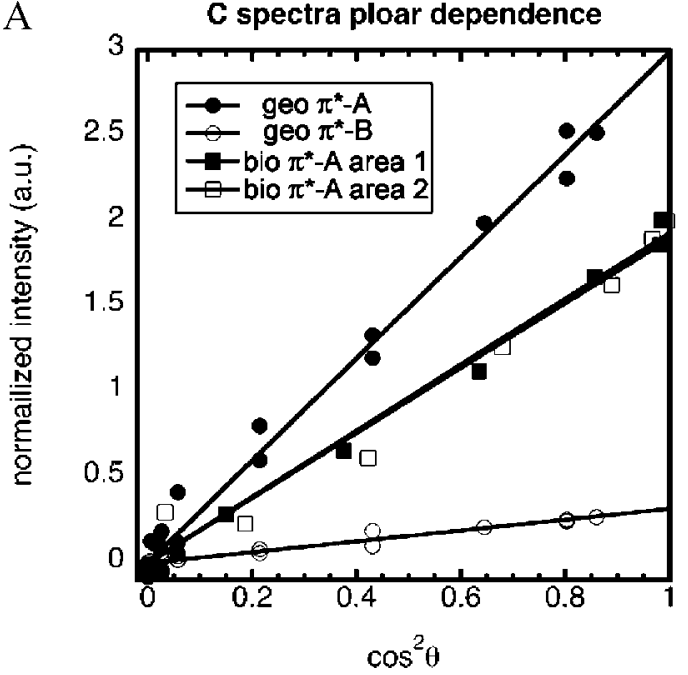

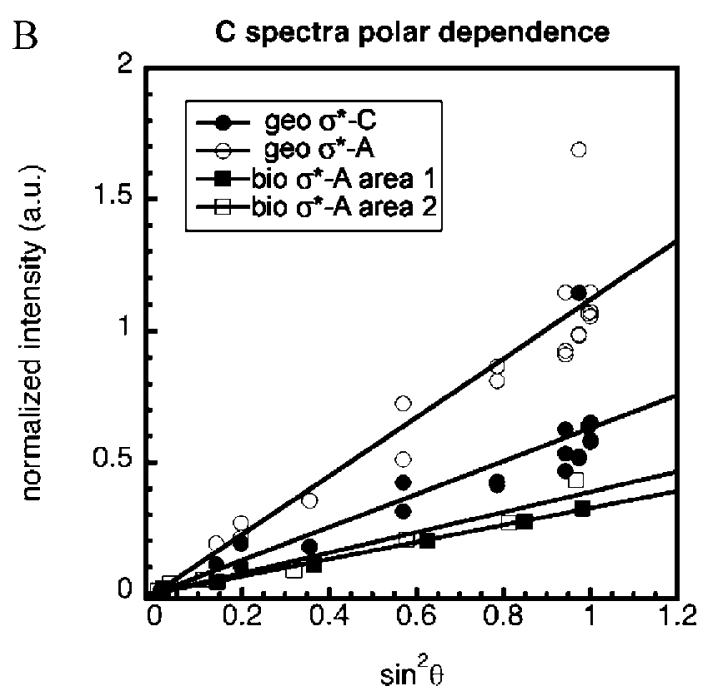

Figure 5. Polar dependence of the carbon XANES peaks. The same data in Figure 3F (STXM) and Figure 4F (FY data) are presented here with explicit $\sin ^{2} \theta$ and $\cos ^{2} \theta$ dependence. For the geologic sample, the polar angle $\theta$ is known. For the biogenic sample, we used the average value of the fitted parameter $\theta_{0}$ and $\theta_{0 \mathrm{~b}}$ as the polar angle. The residue intensities $I_{\mathrm{o}}$ are removed so that all data can be fitted to the linear form $y=k x$. Notice the similarity of the $\pi^{*}$-A peak intensities from both areas on the biosample, and the difference between geo- $\pi^{*}$-A and geo- $\pi^{*}$-B data.

an example of the $\mathrm{PIC}^{9}$ mentioned above. We acquired a series of images at different EPU polarization angles, and extracted spectra from two regions of interest, labeled area 1 and area 2 in Figure 3C. The corresponding spectra are reported in Figure $3 \mathrm{D}, \mathrm{E}$. From these spectra it is evident that the $\pi^{*}$ and $\sigma^{*}$ main peak intensities, at 290.3 and $302.2 \mathrm{eV}$, respectively, are 
TABLE 1: All Peaks Observed in Biogenic and Geologic Aragonite in Figure 2 and Their Assignment to Specific Bonds ${ }^{a}$

\begin{tabular}{|c|c|c|c|c|c|}
\hline $\begin{array}{l}\text { peak } \\
\text { energy }\end{array}$ & $\begin{array}{c}\text { TEY } \\
\text { aragonite }\end{array}$ & $\begin{array}{c}\text { FY } \\
\text { aragonite }\end{array}$ & $\begin{array}{c}\text { STXM } \\
\text { aragonite }\end{array}$ & $\begin{array}{l}\text { STXM } \\
\text { nacre }\end{array}$ & peak assignment \\
\hline 285.0 & $\sqrt{ }$ & & & $\sqrt{ }$ & $\mathrm{C}=\mathrm{C}$ in organic molecules \\
\hline 286.7 & $\sqrt{ }$ & & & $\sqrt{ }$ & $\mathrm{C}-\mathrm{H}$ in organic molecules \\
\hline 290.3 & $\sqrt{ }$ & $\sqrt{ }$ & $\sqrt{ }$ & $\sqrt{ }$ & $\begin{array}{l}\text { CO } \pi^{*} \text {-A in carbonate, correlated with peaks at } 294.2 \text { and } 298.3 \\
\text { eV }\end{array}$ \\
\hline 294.2 & $\sqrt{ }$ & $\sqrt{ }$ & $\sqrt{ }$ & $\sqrt{ }$ & CO $\pi^{*}-\mathrm{B}$ carbonate, $\sigma^{*}$ in carboxylate \\
\hline 300.2 & $\sqrt{ }$ & $\sqrt{ }$ & $\sqrt{ }$ & $\sqrt{ }$ & $\mathrm{CO} \sigma^{*}-\mathrm{B}$ in carbonate \\
\hline 302.2 & $\sqrt{ }$ & $\sqrt{ }$ & $\sqrt{ }$ & $\sqrt{ }$ & $\mathrm{CO} \sigma^{*}-\mathrm{A}$ in carbonate(correlated with peaks at 295.9 and $300.2 \mathrm{eV}$ ) \\
\hline
\end{tabular}

${ }^{a}$ The three peaks in the bold rows are correlated in polarized light angular dependence curves.

anticorrelated: the $\sigma^{*}$ peak intensity increases with the polarization angle, while the $\pi^{*}$ decreases. The polarization dependence of the two peaks in both areas is reported in Figure $3 \mathrm{~F}$, and these are well fitted by $\cos ^{2}$ and $\sin ^{2}$ curves, for the $\pi^{*}$-A and $\sigma^{*}$-A, respectively. This is in close agreement with theory ${ }^{16}$ and prior observation of macroscopic crystals rotated in front of the beam. ${ }^{9}$ As expected, there is no detectable difference in the polarization dependence of the main $\pi^{*}$ and $\sigma^{*}$ peaks between biogenic and geologic aragonite.

In Figure 4, we report polarization-dependence maps, acquired with STXM on nacre thinned by tripod polishing. Notice that the intensities of the main $\mathrm{CO}$ bond $\pi^{*}$ and $\sigma^{*}$ peaks are anticorrelated from the imaging point of view as well: a crystal that appears bright in the $\pi^{*}$ map is dark in the $\sigma^{*}$ map.

The contrast observed in Figure 4A is consistent with the nacre sample position, with the growth direction at $21^{\circ}$ from the surface normal. As seen from the footprint of the rocking curve obtained from red abalone nacre and $\mathrm{XRD},{ }^{9}$ the $c$-axis spread is $22^{\circ}$. From microbeam XRD (data not shown) a similar spread in $c$-axis orientation is observed in immediately adjacent tablets. The angle between the $c$-axis and the polarization vector, therefore, can be as large as $\theta=90^{\circ}$ and as small as $\theta=90^{\circ}$ $-\left(21^{\circ}+11^{\circ}\right)=58^{\circ}$. This corresponds to a $\cos ^{2} \theta=0$ and 0.28 , respectively. This large spectral peak intensity variation justifies the large contrast observed in Figure 4A in adjacent tablets.

In Figure 4 we also report maps for the minor peaks, and find that the polarization-dependence maps at 294.2 and 298.3 $\mathrm{eV}$ correlate in intensity and contrast with the main $\pi^{*}$-A map. These three maps with correlated contrast are reported in Figure 4A-C. We therefore assign the minor 294.2 and $298 \mathrm{eV}$ peaks to $\mathrm{CO} \pi^{*}$ resonances. On the basis of this evidence, we labeled these peaks and the corresponding maps $\pi^{*}$-B and $\pi^{*}-\mathrm{C}$.

Two other polarization-dependence maps are correlated in intensity: the one at $295.9 \mathrm{eV}$ and the one at $300.2 \mathrm{eV}$. Because the $\mathrm{CO}$ bond $\sigma^{*}$-B and $\sigma^{*}$-A resonances at 300.2 and $302.2 \mathrm{eV}$ are well established, from the correlation with these, we deduce that the $295.9 \mathrm{eV}$ peak must also be due to a $\mathrm{CO} \sigma^{*}$ resonance, and labeled this $\sigma^{*}$-C.

The assignment of all carbonate peaks into two groups of molecular orbitals is consistent with the vector-type molecular orbital description discussed by Stöhr, ${ }^{16}$ which applies to both single and double bonds, and is therefore appropriate for carbonate $\mathrm{CO}$ bonds. Two independent experimental observations reported here, the anticorrelation of $\pi^{*}$ and $\sigma^{*}$ peak intensities at each polarization angle (Figures $3 \mathrm{~F}$ and $4 \mathrm{~F}$ ), and the inversion of contrast in polarization-dependence maps (e.g., Figure 4A,D) demonstrate that the $\pi^{*}$ and $\sigma^{*}$ antibonding molecular orbitals, probed by the XANES $\pi^{*}$ and $\sigma^{*}$ peaks, are indeed perpendicular to each other.
In the STXM experiment the intensity of the minor peaks was too low to produce angular dependence curves as those of the main peaks in Figure 3F. Thus, to corroborate the assignment of two of the minor peaks to $\pi^{*}$ and $\sigma^{*}$ orbitals, we further analyzed their angular dependence with FY in a macroscopic single crystal of aragonite. The angular dependence of the minor peaks is reported in Figure $4 \mathrm{~F}$, and compared with the main $\pi^{*}$-A and $\sigma^{*}$-A peaks. It is evident from this plot that the 294.2 and $290.3 \mathrm{eV}$ peaks respond identically to polarization, as does the other peak at $298.3 \mathrm{eV}$, omitted for clarity from Figure 4F, as it is very similar in intensity and polarization dependence to that at $294.2 \mathrm{eV}$. The three peaks at $295.9 \mathrm{eV}, 300.2$, and 302.2 $\mathrm{eV}$ peaks also respond identically to polarization, and are phase shifted by $90^{\circ}$ from the previous peaks. The unassigned peaks at 294.2 and $295.9 \mathrm{eV}$ must therefore be $\pi^{*}$ and $\sigma^{*}$ peaks, respectively. Hence they are labeled as $\pi^{*}$-B and $\sigma^{*}$ - C, respectively.

In order to estimate the data quality, we tested the linear dependence of peak intensities as a function of $\cos ^{2} \theta$ and $\sin ^{2}$ $\theta$, for the same peak intensities reported in Figures $3 \mathrm{~F}$ and $4 \mathrm{~F}$. These plots are presented in Figure 5. The linear fit goodness is informative, while the slopes of the linear fits are not. The slopes represent the maximum variations in the peak intensities while one varies the polar angle from $0^{\circ}$ to $90^{\circ}$. Notice that the slope depends significantly on experimental conditions, that is, for the same peak, STXM and FY give different slopes. This is because the physical processes generating the detected signals are different for STXM and FY (and for TEY), thus the absolute magnitude of the slopes cannot be compared directly for different data acquisition techniques. Nevertheless, some observations can be made from these plots. The similarity of the $\pi^{*}$-A peak intensities from both areas on the biosample shows the reproducibility of the STXM data. The geo- $\pi^{*}$-A and geo$\pi^{*}$-B slopes are very different, although these FY data were acquired simultaneously from the same spot on the sample. Clearly $\pi^{*}$-B is less intense, and therefore varies less than $\pi^{*}$ A. This is why we called this and other minor peaks $-\mathrm{B}$ and $-\mathrm{C}$. The overall data trends and their linearity demonstrate that the peak assignment proposed above is robust.

\section{Discussion}

The observation and assignment of the minor peaks in aragonite is particularly relevant in biomineralization. We have observed both the major and minor carbonate peaks in a variety of biomineral samples, including cowrie, conch, pearl, and fossilized wood, along with geologic and synthetic calcite. In previous work the minor peaks were found to be dramatically altered upon biomineral formation, ${ }^{7,9,19}$ and this result was confirmed in the present work, when comparing biogenic nacre 
and geologic aragonite. We stress that this is not the result of a dichroic effect, but of a biological effect. In fact the intensity variations observed in the minor peaks are not correlated with variations in the main $\pi^{*}$ and $\sigma^{*}$ peaks, as would be expected if this were a dichronic effect. Only the minor peaks are affected, while the main peaks are unaltered, in this work on nacre, in that of Metzler et al. ${ }^{7}$ and that of Benzerara et al. ${ }^{19}$ Interaction with organic molecules must be affecting the intensity of the minor peaks. In nacre proteins, glycoproteins and polysaccharides are present in high concentration, up to $5 \%$ by either mass or volume, ${ }^{28}$ and many proteins are known to be intracrystalline in aragonite tablets. ${ }^{29,30}$ In the Lake Van microbialites analyzed by Benzerara et al., ${ }^{19}$ the difference between intra- and intercellular aragonite spectra is in agreement with that observed here between bio- and geo-aragonite, respectively. Although the role of microbes in microbialite formation is highly debated, one safe assumption can be made: there must be higher concentration of organics intracellularly than intercellularly. When both intra- and intercellular spaces become mineralized with aragonite, therefore, aragonite binds more organics in cells than between cells. Benzerara et al. show a direct comparison of intra- and intercellular aragonite XANES spectra at the carbon K-edge, and observe striking differences due to organics in the pre-290 eV peaks. They do not comment about the minor peaks discussed here, but their results are in perfect agreement with ours, when considering their intracellular aragonite as biogenic (that is, formed in the presence of high concentration of organics), and intercellular aragonite as geologic.

Pokroy et al. described structural distortions between biogenic and geologic aragonite. ${ }^{13-15}$ We do not expect such small distortions to affect XANES spectra. Moreover, crystal structure distortion should affect most dramatically the main aragonite peaks. In all biomineral systems thus far analyzed, however, the minor peaks are affected upon biomineral formation, and not the main ones. Although we do not yet know why upon biomineral formation the minor peaks should be more affected than the main ones, we propose that this is a result of binding of organic molecules to carbonates.

\section{Conclusions}

We have shown that the peak locations and polarization dependence of the carbon XANES spectra of biogenic and geologic aragonite are similar to each other, with the only differences being the relative intensities of minor peaks. We have for the first time analyzed in depth the presence and polarization behavior of four minor peaks in aragonite and nacre, and we assigned them to $\mathrm{CO} \pi^{*}$ and $\sigma^{*}$ resonances. These results provide the basis for the spectroscopic, microscopic, and polarization dependent behavior of aragonitic biominerals.

Acknowledgment. We thank Paul Voyles and Ye Zhu for their help with tripod polishing, and Matthew Marcus for the geologic aragonite samples. We are grateful to Adam Hitchcock for his valuable suggestions on peak assignment. This work was supported by NSF awards PHY-0523905 and CHE\&DMR0613972, DoE award DE-FG02-07ER15899 and UW Vilas and Hamel Awards to PUPAG. The experiments were performed at the ALS, supported by DoE under contract DE-AC02-
05CH11231, and at the UW-SRC, supported by NSF award DMR-0537588.

\section{References and Notes}

(1) Lowenstam, H. A.; Weiner, S. On Biomineralization; Oxford University Press: New York, 1989.

(2) Mann, S. Biomineralization: Principles and Concepts in Bioinorganic Materials Chemistry; Oxford University Press: New York, 2001.

(3) Currey, J. D. Proc. R. Soc. London, Ser. B 1977, 196, 443.

(4) Addadi, L.; Joester, D.; Nudelman, F.; Weiner, S. Chemistry 2006, 12, 980 .

(5) Weiss, I. M.; Renner, C.; Strigl, M. G.; Fritz, M. Chem. Mater. 2002, 14, 3252.

(6) Rousseau, M.; Lopez, E.; Stempfle, P.; Brendle, M.; Franke, L.; Guette, A.; Naslain, R.; Bourrat, X. Biomaterials 2005, 26, 6254.

(7) Metzler, R. A.; Kim, I. W.; Delak, K.; Evans, J. S.; Zhou, D.; Beniash, E.; Wilt, F.; Abrecht, M.; Chiou, J. W.; Guo, J.; Coppersmith, S. N.; Gilbert, P. U. P. A. Langmuir 2008, 24, 2680.

(8) Metzler, R. A.; Abrecht, M.; Olabisi, R. M.; Ariosa, D.; Johnson, C. J.; Frazer, B. H.; Coppersmith, S. N.; Gilbert, P. U. P. A. Phys. Rev. Lett. 2007, 98, 268102.

(9) Metzler, R. A.; Zhou, D.; Abrecht, M.; Chiou, J. W.; Guo, J. H.; Ariosa, D.; Coppersmith, S. N.; Gilbert, P. U. P. A. Phys. Rev. B 2008, 77 , 064110.

(10) Frazer, B. H.; Girasole, M.; Wiese, L. M.; Franz, T.; De Stasio, G. Ultramicroscopy 2004, 99, 87.

(11) Kilcoyne, A. L. D.; Tyliszczak, T.; Steele, W. F.; Fakra, S.; Hitchcock, P.; Franck, K.; Anderson, E.; Harteneck, B.; Rightor, E. G.; Mitchell, G. E.; Hitchcock, A. P.; Yang, L.; Warwick, T.; Ade, H. J. Synchrotron Radiat. 2003, 10, 125.

(12) Myneni, S. C. B. Soft X-ray spectroscopy and spectromicroscopy studies of organic molecules in the environment. In Applications of Synchrotron Radiation in Low-Temperature Geochemistry and Environmental Science; Fenter, P., Rivers, M., Sturchio, N., Sutton, S., Eds.; Mineralogical Society of America: Washington, DC, 2002; Vol. 49; pp 485.

(13) Pokroy, B.; Fieramosca, J. S.; Von Dreele, R. B.; Fitch, A. N.; Caspi, E. N.; Zolotoyabko, E. Chem. Mater. 2007, 19, 3244.

(14) Pokroy, B.; Fitch, A. N.; Zolotoyabko, E. Cryst. Growth Des. 2007, 7, 1580 .

(15) Pokroy, B.; Quintana, J. P.; Caspi, E. N.; Berner, A.; Zolotoyabko, E. Nat. Mater. 2004, 3, 900.

(16) Stöhr, J. NEXAFS Spectroscopy; Springer: Berlin, 1996.

(17) Madix, R. J.; Solomon, J. L.; Stohr, J. Surf. Sci. 1988, 197, L253.

(18) Reeder, R. J. Carbonates: Mineralogy and Chemistry; Mineralogical Society of America: Washington, DC, 1983; Vol. 11.

(19) Benzerara, K.; Menguy, N.; Lopez-Garcia, P.; Yoon, T.-H.; Kazmierczak, J.; Tyliszczak, T.; Guyot, F.; Brown, G. E. Proc. Natl. Acad. Sci. 2006, 103, 9440.

(20) http://henke.lbl.gov/optical_constants/atten2.html.

(21) De Stasio, G.; Frazer, B. H.; Gilbert, B.; Richter, K. L.; Valley, J. W. Ultramicroscopy 2003, 98, 57.

(22) Lawrence, J. R.; Swerhone, G. D. W.; Leppard, G. G.; Araki, T.; Zhang, X.; West, M. M.; Hitchcock, A. P. Appl. Environ. Microbiol. 2003, 69, 5543.

(23) Kaznacheyev, K.; Osanna, A.; Jacobsen, C.; Plashkevych, O.; Vahtras, O.; Ågren, H.; Carravetta, V.; Hitchcock, A. P. J. Phys. Chem. A 2002, 106, 3153

(24) de Leeuw, N. H.; Parker, S. C. J. Phys. Chem. B 1998, 102, 2914.

(25) Magdans, U.; Gies, H.; Torrelles, X.; Rius, J. Eur. J. Miner. 2006,

$18,83$.

(26) van Aken, P. A.; Lauterbach, S. Phys. Chem. Miner. 2004, 30, 469.

(27) Templeton, D. H.; Templeton, L. K. Acta Crystallogr., Sect. A 1997, 53,352 .

(28) Jackson, A. P.; Vincent, J. F. V.; Turner, R. M. Proc. R. Soc. B 1988, $234,415$.

(29) Addadi, L.; Berman, A.; Weiner, S. Intracrystalline proteins from a sea urchin and a mollusk: A comparison. In Mechanisms and Phylogeny of Mineralization in Biological Systems; Suga, S., Nakahara, H., Eds.; Springer-Verlag: Tokyo,1991; pp 29.

(30) Levi-Kalisman, Y.; Falini, G.; Addadi, L.; Weiner, S. J. Struct. Biol. 2001, 135, 8.

JP803176Z 